\title{
Optimal stopping with delayed information
}

\author{
Bernt Øksendal ${ }^{1,2}$
}

Revised in May 2005

1 Center of Mathematics for Applications (CMA) Department of Mathematics, University of Oslo P.O. Box 1053 Blindern, N-0316, Oslo, Norway and

2 Norwegian School of Economics and Business Administration, Helleveien 30, N-5045, Bergen, Norway

\begin{abstract}
We study a general optimal stopping problem for a strong Markov process in the case when there is a time lag $\delta>0$ from the time $\tau$ when the decision to stop is taken (a stopping time) to the time $\tau+\delta$ when the system actually stops. Equivalently, we impose the constraint that the admissible times for stopping are stopping (Markov) times with respect to the delayed flow of information. It is shown that such a problem can be reduced to a classical optimal stopping problem by a simple transformation. The results are applied

(i) to find the optimal time to sell an asset

(ii) to solve an optimal resource extraction problem, in both cases under delayed information flow.
\end{abstract}

MSC (200): 93C41, 93E20, 60J25, 91B28

Key words: Optimal stopping, delayed information flow, strong Markov processes, optimal time to sell, optimal time to stop resource extraction.

\section{Introduction}

Let $Y(t)$ be a strong Markov process in $\mathbb{R}^{k}$ on a filtered probability space $\left(\Omega, \mathcal{F},\left\{\mathcal{F}_{t}\right\}_{t \geq 0}\right.$, $\left\{P^{y}\right\}_{y \in \mathbb{R}^{n}}$ ), where $P^{y}$ is the probability measure giving the law of $\{Y(t)\}_{t \geq 0}$ when $Y(0)=$ $y \in \mathbb{R}^{k}$. Let $\delta \geq 0$ be a fixed constant. In this paper we consider optimal stopping problems of the form

$$
\Phi_{\delta}(y):=\sup _{\alpha \in \mathcal{T}_{\delta}} E^{y}\left[\int_{0}^{\alpha} f(Y(t)) d t+g(Y(\alpha))\right]
$$


where $E^{y}$ denotes expectation with respect to $P^{y}$ and $f: \mathbb{R}^{k} \rightarrow \mathbb{R}, g: \mathbb{R}^{k} \rightarrow \mathbb{R}$ are given continuous functions such that

$$
E^{y}\left[\int_{0}^{\alpha}|f(Y(t))| d t+|g(Y(\alpha))|\right]<\infty \quad \text { for all } \alpha \in \mathcal{T}_{\delta}
$$

where we interpret $g(Y(\alpha))$ as 0 if $\alpha=\infty$. Here $\mathcal{T}_{\delta}$ is the set of $\delta$-delayed stopping times, defined as follows

Definition 1.1 A function $\alpha: \Omega \rightarrow[\delta, \infty]$ is called a $\delta$-delayed stopping time if

$$
\{\omega ; \alpha(\omega) \leq t\} \in \mathcal{F}_{t-\delta} \quad \text { for all } t \geq \delta
$$

or, equivalently,

$$
\{\omega ; \alpha(\omega) \leq s+\delta\} \in \mathcal{F}_{s} \quad \text { for all } s \geq 0
$$

The set of all $\delta$-delayed stopping times is denoted by $\mathcal{T}_{\delta}$.

In other words, if we interpret $\alpha(\omega)$ as the time to stop, then $\alpha \in \mathcal{I}_{\delta}$ if the decision whether or not to stop at or before time $t$ is based on the information represented by $\mathcal{F}_{t-\delta}$. In particular, if $\delta=0$ then $\mathcal{T}_{\delta}=\mathcal{T}_{0}$ is the family of classical stopping times and (1.1) becomes the classical optimal stopping problem, discussed in many texts (see e.g. [Ø, Ch. 10]).

In the delayed case problem (1.1) models the situation when there is a delay $\delta>0$ in the flow of information available to the agent searching for the optimal time to stop. An alternative way of stating this, is that there is a delay $\delta>0$ from the decided stopping time $\tau \in \mathcal{T}_{0}$ (based on the complete current information available from the system) to the time $\alpha=\tau+\delta \in \mathcal{T}_{\delta}$ when the system actually stops. This new formulation is based on the following simple observation:

Lemma 1.2 (i) $\quad \tau \in \mathcal{T}_{0} \Longleftrightarrow \alpha:=\tau+\delta \in \mathcal{T}_{\delta}$

(ii) $\alpha \in \mathcal{T}_{\delta} \Longleftrightarrow \tau:=\alpha-\delta \in \mathcal{T}_{0}$

Proof. It suffices to prove (i).

First, assume $\tau \in \mathcal{T}_{0}$. Then, for $t \geq \delta$,

$$
\{\omega ; \tau(\omega)+\delta \leq t\}=\{\omega ; \tau(\omega) \leq t-\delta\} \in \mathcal{F}_{t-\delta}
$$

and hence $\alpha:=\tau+\delta \in \mathcal{T}_{\delta}$.

Conversely, if $\alpha:=\tau+\delta \in \mathcal{T}_{\delta}$ then

$$
\{\omega ; \tau(\omega) \leq t\}=\{\omega ; \tau(\omega)+\delta \leq t+\delta\}=\{\omega ; \alpha(\omega) \leq t+\delta\} \in \mathcal{F}_{(t+\delta)-\delta}=\mathcal{F}_{t}
$$

and hence $\tau \in \mathcal{T}_{0}$. 
Remark 1.3 In view of this result we see that it is possible to give another interpretation of problem (1.1), namely

$$
\Phi_{\delta}(y)=\sup _{\tau \in \mathcal{T}_{0}} E^{y}\left[\int_{0}^{\tau+\delta} f(Y(t)) d t+g(Y(\tau+\delta))\right]
$$

In this formulation the problem appears as an optimal stopping problem over classical stopping times $\tau \in \mathcal{T}_{0}$, but with delayed effect of the stopping: If the stopping time $\tau \in \mathcal{T}_{0}$ is chosen, then the system itself is stopped at time $\tau+\delta$, i.e. after a delay $\delta>0$.

Note that $\mathcal{T}_{\delta} \subset \mathcal{T}_{0}$ for $\delta>0$ and hence

$$
\Phi_{\delta}(y) \leq \Phi_{0}(y)
$$

and we can interpret $\Phi_{0}(y)-\Phi_{\delta}(y)$ as the loss of value due to the delay of information.

In this paper we show that the delayed optimal stopping problem (1.1) can be reduced to a classical optimal stopping problem by a simple transformation (Theorem 2.1).

We call $\alpha^{*} \in \mathcal{T}_{\delta}$ an optimal stopping time for the problem (1.1) if

$$
\Phi_{\delta}(y)=E^{y}\left[\int_{0}^{\alpha^{*}} f(Y(t)) d t+g\left(Y\left(\alpha^{*}\right)\right)\right] .
$$

This paper may be regarded as a partial extension of [AK2], where the geometric Brownian motion case is studied and solved (see Example 3.1), with a more general (Markovian) delay $\delta(X) \leq 0$. See also [AK1]. For a related type of problem involving impulse control with delivery lags see [BS].

\section{Optimal stopping with $\delta$-delayed information}

We are now ready to state and prove the main result of this paper:

Theorem 2.1 a) Consider the two optimal stopping problems:

$$
\begin{aligned}
& \Phi_{\delta}(y):=\sup _{\alpha \in \mathcal{T}_{\delta}} E^{y}\left[\int_{0}^{\alpha} f(Y(t)) d t+g(Y(\alpha))\right] \\
& \tilde{\Phi}(y):=\sup _{\tau \in \mathcal{T}_{0}} E^{y}\left[\int_{0}^{\tau} f(Y(t)) d t+\tilde{g}_{\delta}(Y(\tau))\right]
\end{aligned}
$$

where

$$
\tilde{g}_{\delta}(y)=E^{y}\left[\int_{0}^{\delta} f(Y(t)) d t+g(Y(\delta))\right]
$$

Then we have

$$
\Phi_{\delta}(y)=\tilde{\Phi}(y) \quad \text { for all } y \in \mathbb{R}^{k}, \delta \geq 0 \text {. }
$$


b) Moreover, $\alpha^{*} \in \mathcal{T}_{\delta}$ is an optimal stopping time for the delayed problem (2.1) if and only if

$$
\alpha^{*}:=\tau^{*}+\delta
$$

where $\tau^{*} \in \mathcal{T}_{0}$ is an optimal stopping time for the non-delayed problem (2.2).

Proof. a) Define

$$
J^{(\alpha)}(y)=E^{y}\left[\int_{0}^{\alpha} f(Y(t)) d t+g(Y(\alpha))\right] ; \quad \alpha \in \mathcal{T}_{\delta},
$$

and

$$
\tilde{J}^{(\tau)}(y)=E^{y}\left[\int_{0}^{\tau} f(Y(t)) d t+\tilde{g}_{\delta}(Y(\tau))\right] ; \quad \tau \in \mathcal{T}_{0} .
$$

Choose $\alpha \in \mathcal{T}_{\delta}$ and put

$$
\tau=\alpha-\delta \in \mathcal{T}_{0}
$$

Then $\alpha=\tau+\delta$ and hence

$$
\begin{aligned}
J^{(\alpha)}(y) & =E^{y}\left[\int_{0}^{\alpha} f(Y(t)) d t+g(Y(\alpha))\right] \\
& =E^{y}\left[\int_{0}^{\tau+\delta} f(Y(t)) d t+g(Y(\tau+\delta))\right] \\
& =E^{y}\left[\int_{0}^{\tau} f(Y(t)) d t+\int_{\tau}^{\tau+\delta} f(Y(t)) d t+g(Y(\tau+\delta))\right] \\
& =E^{y}\left[\int_{0}^{\tau} f(Y(t)) d t+E^{y}\left[\theta_{\tau}\left\{\int_{0}^{\delta} f(Y(t)) d t+g(Y(\delta))\right\}\right]\right],
\end{aligned}
$$

where $\theta_{\tau}$ is the shift operator, defined by

$$
\theta_{\tau}\{h(Y(s))\}=h(Y(\tau+s)) \text { for } s \geq 0, \text { for all measurable } h: \mathbb{R}^{k} \longrightarrow \mathbb{R},
$$

and we have used that

$$
\theta_{\tau}\left(\int_{0}^{\delta} f(Y(t)) d t\right)=\int_{\tau}^{\tau+\delta} f(Y(t)) d t
$$

We refer to $[\mathrm{BG}]$ for more information about Markov processes. By the strong Markov property we now get from (2.7) that

$$
\begin{aligned}
J^{(\alpha)}(y) & =E^{y}\left[\int_{0}^{\tau} f(Y(t)) d t+E^{y}\left[\theta_{\tau}\left\{\int_{0}^{\tau} f(Y(t)) d t+g(Y(\delta))\right\} \mid \mathcal{F}_{\tau}\right]\right] \\
& =E^{y}\left[\int_{0}^{\tau} f(Y(t)) d t+E^{Y(\tau)}\left[\int_{0}^{\delta} f(Y(t)) d t+g(Y(\delta))\right]\right] \\
& =E^{y}\left[\int_{0}^{\tau} f(Y(t)) d t+\tilde{g}_{\delta}(Y(\tau))\right]=\tilde{J}^{(\tau)}(y) .
\end{aligned}
$$


Hence, by Lemma 1.2 (ii),

$$
\begin{aligned}
\Phi_{\delta}(y) & =\sup _{\alpha \in \mathcal{T}_{\delta}} J^{(\alpha)}(y)=\sup _{\alpha-\delta \in \mathcal{T}_{0}} J^{(\alpha)}(y) \\
& =\sup _{\alpha-\delta \in \mathcal{T}_{0}} \tilde{J}^{(\alpha-\delta)}(y)=\sup _{\tau \in \mathcal{T}_{0}} \tilde{J}^{(\tau)}(y)=\tilde{\Phi}(y),
\end{aligned}
$$

as claimed.

b) Suppose $\tau^{*} \in \mathcal{T}_{0}$ is optimal for (2.2). Define

$$
\alpha^{*}:=\tau^{*}+\delta .
$$

Then $\alpha^{*} \in \mathcal{T}_{\delta}$ by Lemma 1.2 and by (2.8) combined with a) we have

$$
J^{\left(\alpha^{*}\right)}(y)=\tilde{J}^{\left(\tau^{*}\right)}(y)=\tilde{\Phi}(y)=\Phi_{\delta}(y) .
$$

Hence $\alpha^{*}$ is optimal for (2.1).

Conversely, if $\alpha^{*} \in \mathcal{T}_{\delta}$ is optimal for (2.1) a similar argument gives that $\tau^{*}:=\alpha^{*}-\delta$ is optimal for (2.2).

\section{Application 1: The optimal time to sell an asset}

In this section we illustrate Theorem 2.1 by solving the following problem:

\section{Example 3.1 (The optimal time to sell an asset)}

This case (without the jump part) was first solved by [AK1], with a more general (Markovian) delay $\delta(X) \geq 0$.

Suppose the value $X(t)$ of an asset at time $t$ is modelled by a geometric Lévy process of the form

$$
d X(t)=X\left(t^{-}\right)\left[\mu d t+\sigma d B(t)+\int_{\mathbb{R}} z \tilde{N}(d t, d z)\right], \quad X(0)=x>0,
$$

where $\mu, \sigma$ and $x$ are constants. Here $B(t)$ and $\eta(t):=\int_{0}^{t} \int_{\mathbb{R}} z \tilde{N}(d s, d z)$ is a Brownian motion and an independent pure jump Lévy process, respectively, where

$$
\tilde{N}(d t, d z)=N(d t, d z)-\nu(d z) d t
$$

is the compensated Poisson random measure of $\eta(\cdot), N(d t, d z)$ is the Poisson random measure of $\eta(\cdot)$ and $\nu(d z)$ is the Lévy measure of $\eta(\cdot)$. We assume that

$$
0 \geq z \geq-1 \text { a.s. } \nu \text {. }
$$


This guarantees that $X(t)$ never jumps down to a negative value. For convenience, we also assume that

$$
E\left[\eta^{2}(t)\right]<\infty \quad \text { for all } t \geq 0 .
$$

Then by the Itô formula for Lévy processes (see e.g. [ØS]) the solution of equation (3.1) is

$$
\begin{aligned}
X(t)= & x \exp \left[\left(\mu-\frac{1}{2} \sigma^{2}\right) t+\sigma B(t)\right. \\
& \left.+\int_{0}^{t} \int_{\mathbb{R}}\{\ln (1+z)-z\} \nu(d z) d s+\int_{0}^{t} \int_{\mathbb{R}} \ln (1+z) \tilde{N}(d s, d z)\right] ; \quad t \geq 0 .
\end{aligned}
$$

We now study the following problem

$$
\Phi_{\delta}(s, x)=\sup _{\alpha \in \mathcal{T}_{\delta}} E^{s, x}\left[e^{-\rho(s+\alpha)}(X(\alpha)-q)\right],
$$

where $E^{s, x}$ denotes expectation with respect to the probability law $P^{s, x}$ of the time-space process

$$
d Y(t)=\left[\begin{array}{c}
d t \\
d X(t)
\end{array}\right] ; \quad Y(0)=\left[\begin{array}{l}
s \\
x
\end{array}\right]
$$

and $\rho>0, q>0$ are constants. We assume that

$$
\rho>\mu \text {. }
$$

One possible interpretation of this problem is that $\Phi_{\delta}(s, x)$ represents the maximal expected discounted net payment obtained by selling the asset at a $\delta$-delayed stopping time ( $\rho$ is the discounting exponent and $q$ is the transaction cost).

It is well-known that in the no delay case $(\delta=0)$ the solution of the problem (3.5) is the following (under some additional assumptions on the Lévy measure $\nu$ ):

$$
\Phi_{0}(s, x)=e^{-\rho s} \Psi_{0}(x)
$$

where

$$
\Psi_{0}(x)=\left\{\begin{array}{lll}
x-q & ; & x \geq x_{0}^{*} \\
C_{0} x^{\lambda} & ; \quad 0<x<x_{0}^{*}
\end{array}\right.
$$

Here $\lambda>1$ is uniquely determined by the equation

$$
-\rho+\mu \lambda+\frac{1}{2} \sigma^{2} \lambda(\lambda-1)+\int_{\mathbb{R}}\left\{(1+z)^{\lambda}-1-\lambda z\right\} \nu(d z)=0,
$$

and $x_{0}^{*}$ and $C_{0}$ are given by

$$
\begin{aligned}
x_{0}^{*} & =\frac{\lambda q}{\lambda-1} \\
C_{0} & =\frac{1}{\lambda}\left(x_{0}^{*}\right)^{1-\lambda} .
\end{aligned}
$$


The corresponding optimal stopping time $\tau^{*} \in \mathcal{T}_{0}$ is

$$
\tau^{*}=\inf \left\{t>0 ; X(t) \geq x_{0}^{*}\right\}
$$

Thus it is optimal to sell at the first time the price $X(t)$ equals or exceeds the value $x_{0}^{*}$. We refer to [ØS, Example 2.5] for details.

To find the solution in the delay case $(\delta>0)$ we note that we have $f=0$ and

$$
g(y)=g(s, x)=e^{-\rho s}(x-q)
$$

Hence, by (2.2),

$$
\begin{aligned}
\tilde{g}_{\delta}(y) & =E^{y}[g(Y(\delta))]=E^{s, x}\left[e^{-\rho(s+\delta)}(X(\delta)-q)\right] \\
& =e^{-\rho(s+\delta)}\left(E^{x}[X(\delta)]-q\right)=e^{-\rho(s+\delta)}\left(x e^{\mu \delta}-q\right) \\
& =e^{-\rho s+\delta(\mu-\rho)}\left(x-q e^{-\mu \delta}\right)=K e^{-\rho s}(x-\tilde{q}),
\end{aligned}
$$

where

$$
K=e^{\delta(\mu-\rho)} \quad \text { and } \quad \tilde{q}=q e^{-\mu \delta}
$$

Thus $\tilde{g}_{\delta}$ has the same form as $g$, so we can apply the results (3.7)-(3.12) to find $\tilde{\Phi}(y)$ and the corresponding optimal $\tau^{*}$ :

$$
\tilde{\Phi}(y)=\tilde{\Phi}(s, x)=e^{-\rho s} \tilde{\Psi}(x)
$$

where

$$
\tilde{\Psi}(x)= \begin{cases}K(x-\tilde{q}) & ; \quad x \geq \tilde{x}^{*} \\ \tilde{C} x^{\lambda} & ; \quad 0<x<\tilde{x}^{*}\end{cases}
$$

with $\lambda$ as in (3.9). Here $\tilde{x}^{*}$ and $\tilde{C}$ are given by

$$
\begin{aligned}
\tilde{x}^{*} & =\frac{\lambda \tilde{q}}{\lambda-1} \\
\tilde{C} & =\frac{1}{\lambda}\left(\tilde{x}^{*}\right)^{1-\lambda} .
\end{aligned}
$$

The corresponding optimal stopping time for problem (2.2) and (2.1), respectively, is

$$
\begin{aligned}
\tilde{\tau}^{*} & =\inf \left\{t>0 ; X(t) \geq \tilde{x}^{*}\right\} \\
\alpha^{*} & =\tilde{\tau}^{*}+\delta .
\end{aligned}
$$

Using Theorem 2.1 we conclude the following: 
Theorem 3.2 The value function $\Phi_{\delta}(y)$ for the delayed optimal stopping problem (3.5) is given by

$$
\Phi_{\delta}(y)=\tilde{\Phi}(y)
$$

where $\tilde{\Phi}$ is as in (3.15)-(3.18). The corresponding optimal stopping time $\alpha^{*} \in \mathcal{T}_{\delta}$ is

$$
\alpha^{*}=\inf \left\{t>0 ; X(t) \geq \tilde{x}^{*}\right\}+\delta \text {. }
$$

Remark 3.3 Assume for example that

$$
\mu>0
$$

Then comparing (3.17) with the non-delayed case (3.10) we see that $\tilde{q}>q$ and hence

$$
\tilde{x}^{*}<x_{0}^{*}
$$

Thus, in terms of the delayed effect of the stopping time formulation (see (1.5)), it is optimal to stop at the first time $t=\tilde{\tau}^{*}$ when $X(t) \geq \tilde{x}^{*}$. This is sooner than in the non-delayed case, because of the anticipation that during the delay time interval $\left[\tau^{*}, \tau^{*}+\delta\right] X(t)$ is likely to increase (since $\mu>0$ ). See Figure 1 .

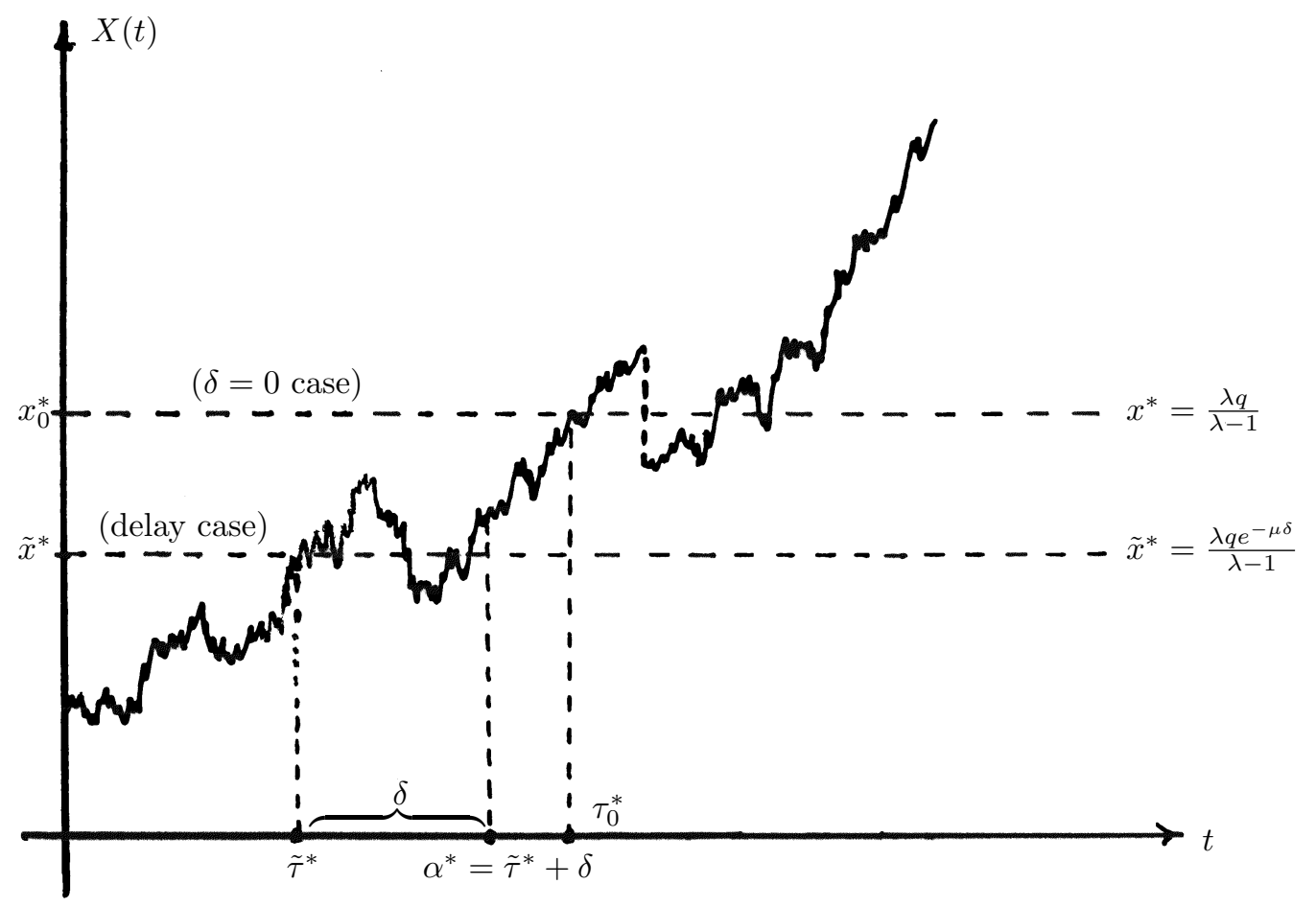

Figure 1. The optimal stopping times for Example $3.1(\mu>0)$ 


\section{Application 2: An optimal resource extraction prob- lem}

In the no delay case the following example was discussed in $[\varnothing]$ (continuous case) and [ØS] (jump diffusion case). Our example models the situation when there is a time lag $\delta>0$ between the decided stopping time $\tau \in \mathcal{T}_{0}$ and the time $\alpha=\tau+\delta \in \mathcal{T}_{\delta}$ when the result of the stopping decision comes into effect.

Example 4.1 (Optimal time to stop resource extraction) Suppose the price $P(t)$ at time $t$ per unit of a resource (oil, gas, ...) is given by

$$
d P(t)=P\left(t^{-}\right)\left[\mu d t+\sigma d B(t)+\int_{\mathbb{R}} z \tilde{N}(d t, d z)\right] ; \quad P(0)=p>0
$$

where, as in Example 3.1, $\mu$ and $\sigma$ are given constants and we assume that $z \geq 0$ a.s. with respect to $\nu$.

Let $Q(t)$ denote the amount of remaining resources at time $t$. As long as the extraction field is open, we assume that the extraction rate is proportional to the remaining amount, i.e.

$$
d Q(t)=-\lambda Q(t) d t ; \quad Q(0)=q>0
$$

where $\lambda>0$ is a known constant.

If we decide to stop the extraction and close the field at a (delayed) stopping time $\alpha \in \mathcal{T}_{\delta}$, then the expected total discounted net profit $J^{\alpha}(s, p, q)$ is assumed to have the form

$$
J^{\alpha}(s, p, q)=E^{(s, p, q)}\left[\int_{0}^{\alpha} e^{-\rho(s+t)}(\lambda P(t) Q(t)-K) d t+\theta e^{-\rho(s+\alpha)} P(\alpha) Q(\alpha)\right]
$$

where $K>0$ is the (constant) running cost rate and $\rho>0, \theta>0$ are other constants. The expectation $E^{(s, p, q)}$ is taken with respect to the probability law $P^{(s, p, q)}$ of the strong Markov process

$$
Y(t):=\left[\begin{array}{l}
s+t \\
P(t) \\
Q(t)
\end{array}\right], \text { which starts at } y=\left[\begin{array}{l}
s \\
p \\
q
\end{array}\right] \text { at time } t=0
$$

The explanation of the quantity $J^{\alpha}(s, p, q)$ in (4.3) is the following:

As long as the field is open (i.e. as long as $t<\alpha$ ) the gross income rate from the production is price times production rate, i.e. $P(t) \lambda Q(t)$. Subtracting the running cost rate $K$ we get the net profit rate

$$
\lambda P(t) Q(t)-K \quad \text { for } 0 \leq t<\alpha .
$$


If the field is closed at time $\alpha$ the net value of the remaining resources is estimated to be $\theta P(\alpha) Q(\alpha)$. Discounting and integrating/adding these quantities and taking expectation we get (4.3).

We want to find the value function $\Phi_{\delta}(s, p, q)$ and the corresponding optimal delayed stopping time $\alpha^{*} \in \mathcal{T}_{\delta}$ such that

$$
\Phi_{\delta}(y)=\Phi_{\delta}(s, p, q)=\sup _{\alpha \in \mathcal{T}_{\delta}} J^{\alpha}(s, p, q)=J^{\alpha^{*}}(s, p, q)
$$

In the case of no delay $(\delta=0)$ it is shown in $[\varnothing \mathrm{S}, \mathrm{p} .158-162]$ that if the following relations between the parameters hold:

$$
0<\theta(\lambda+\rho-\mu)<\lambda
$$

then the optimal stopping time $\tau_{0}^{*} \in \mathcal{T}_{0}$ is

$$
\tau_{0}^{*}=\inf \left\{t>0 ; P(t) Q(t) \leq w_{0}^{*}\right\},
$$

where

$$
w_{0}^{*}=\frac{\left(-r_{2}\right) K(\lambda+\rho-\mu)}{\left(1-r_{2}\right) \rho(\lambda-\theta(\lambda+\rho-\mu))},
$$

$r_{2}<0$ being the negative solution of the equation

$$
h(r):=-\rho+(\mu-\lambda) r+\frac{1}{2} \sigma^{2} r(r-1)+\int_{\mathbb{R}}\left\{(1+z)^{r}-1-r z\right\} \nu(d z)=0
$$

In this case we have

$$
f(y)=f(s, p, q)=e^{-\rho s}(\lambda p q-K)
$$

and

$$
g(y)=g(s, p, q)=\theta e^{-\rho s} p q
$$

Thus

$$
\begin{aligned}
\tilde{g}_{\delta}(y) & =E^{y}\left[\int_{0}^{\delta} e^{-\rho(s+t)}(\lambda P(t) Q(t)-K) d t\right]+E^{y}\left[\theta e^{-\rho(s+\delta)} P(\delta) Q(\delta)\right] \\
& =\int_{0}^{\delta} e^{-\rho(s+t)}(\lambda E[P(t) Q(t)]-K) d t+\theta e^{-\rho(s+\delta)} E^{y}[P(\delta) Q(\delta)] \\
& =\int_{0}^{\delta} e^{-\rho(s+t)}\left(\lambda p q e^{(\mu-\lambda) t}-K\right) d t+\theta e^{-\rho(s+\delta)} p q e^{(\mu-\lambda) \delta}
\end{aligned}
$$




$$
\begin{aligned}
& =e^{-\rho s}\left[\left\{(\lambda+\rho-\mu)^{-1} \lambda\left(1-e^{-(\lambda+\rho-\mu) \delta}\right)+\theta e^{-(\lambda+\rho-\mu) \delta}\right\} p q-\frac{K}{\rho}\left(1-e^{-\rho \delta}\right)\right] \\
& =e^{-\rho s}\left[F_{1} p q-F_{2}\right], \quad \text { where } \\
& F_{1}=(\lambda+\rho-\mu)^{-1} \lambda\left(1-e^{-(\lambda+\rho-\mu) \delta}\right)+\theta e^{-(\lambda+\rho-\mu) \delta} \\
& \text { and } \\
& F_{2}=\frac{K}{\rho}\left(1-e^{-\rho \delta}\right)
\end{aligned}
$$

Therefore, according to Theorem 2.1 we have

$$
\Phi_{\delta}(y)=\sup _{\tau \in \mathcal{T}_{0}} E^{y}\left[\int_{0}^{\tau} e^{-\rho(s+t)}(\lambda P(t) Q(t)-K) d t\right]+E^{y}\left[e^{-\rho(s+\tau)}\left(F_{1} P(\tau) Q(\tau)+F_{2}\right)\right]
$$

The method used in [ØS] to provide the solution (4.7)-4.9) in the no delay case can easily be modified to find the optimal stopping time $\tau^{*}$ for the problem (4.13). The result is

$$
w_{\delta}^{*}=\frac{\left(-r_{2}\right) K(\lambda+\rho-\mu) e^{(\lambda-\mu) \delta}}{(1-r) \rho[\lambda-\theta(\lambda+\rho-\mu)]}=w_{0}^{*} e^{(\lambda-\mu) \delta}
$$

We have proved:

Theorem 4.2 The optimal stopping time $\alpha^{*} \in \mathcal{T}_{\delta}$ for the delayed optimal stopping problem is

$$
\alpha^{*}=\tau_{\delta}^{*}+\delta
$$

where

$$
\tau_{\delta}^{*}=\inf \left\{t>0 ; P(t) Q(t) \leq w_{\delta}^{*}\right\}
$$

with $w_{\delta}^{*}$ given by $(4.14)$.

Remark 4.3 Note that the threshold $w_{\delta}^{*}$ for the decision to close down in the case of a time lag in the action only differs from the corresponding threshold $w_{0}^{*}$ in the no delay case by the factor $e^{(\lambda-\mu) \delta}$.

Assume, for example, that $\lambda>\mu$. Then we should decide to stop sooner in the delay case than in the no delay case, because of the anticipation that $P(t) Q(t)$ will probably decrease during the extra time $\delta$ it takes before the closing down actually takes place.

\section{References}

[AK1] L. H. R. Alvarez and J. Keppo: The impact of delivery lags on irreversible investment demand under uncertainty. Manuscript March 1998. 
[AK2] L. H. R. Alvarez and J. Keppo: The impact of delivery lags on irreversible investment under uncertainty. European J. Operational Research 136 (2002), 173-180.

[BG] R. M. Blumenthal and R. K. Getoor: Markov Processes and Potential Theory. Academic Press 1968.

[BS] A. Bar-Ilan and A. Sulem: Explicit solution of inventory problems with delivery lags. Math. Operations Research 20 (1995), 709-720.

[Ø] B. Øksendal: Stochastic Differential Equations. 6th edition. Springer-Verlag 2003.

[ØS] B. Øksendal and A. Sulem: Applied Stochastic Control of Jump Diffusions. SpringerVerlag 2004.

[S] A: Shiryaev: Optimal Stopping Rules. Springer-Verlag 1978. 